\title{
Campo científico y redes de coautoría en la psiquiatría. La producción científica psiquiátrica mexicana sobre el trastorno de la personalidad
}

\author{
Liliana Ramírez Ruiz¹ - Universidad Iberoamericana/Universidad Nacional \\ Autónoma de México.
}

\begin{abstract}
Resumen
En el presente artículo se realiza un análisis de la producción científica generada por investigadores adscritos a instituciones mexicanas sobre el concepto psiquiátrico del "trastorno de la personalidad". La muestra de la investigación contempla las publicaciones en revistas que aparecen en la base de datos Scopus desde el año de 1970 hasta la fecha. A partir del concepto de campo del sociólogo francés Pierre Bourdieu se interpretan mapas realizados con el software para el análisis de redes sociales Pajek, que situan las relaciones estructurales de la producción científica en el tema por coautorías, coautorías por países, productividad y relacionan la posición de las Universidades e Instituciónes de procedencia. Al partir de dos principios de observación, redes y campos, se sostiene que el análisis de la sociología de la ciencia (fundamentado en dichos principios) puede ser fuertemente sustentado.
\end{abstract}

Palabras clave: trastorno de la personalidad, campo, redes de coautoría.

\begin{abstract}
This paper presents an analysis of the scientific literature on the psychiatric concept of "personality disorder" by Mexican researchers. The research sample includes publications in journals that appear in Scopus database since 1970 to date. Based on the concept of field by French sociologist Pierre Bourdieu we interpret maps made with the software for social network analysis Pajek. We locate structural relationships in the scientific production by coauthorship, coauthorship by country, productivity and relating the position of Universities and Institutions of origin. By adopting two principles of observation, fields and networks, it is argued that analysis of the sociology of science is strongly supported.
\end{abstract}

Key words: personality disorder, field, co-authorship networks.

\footnotetext{
${ }^{1}$ Estudiante de la Maestría en Sociología de la Universidad Iberoamericana, Asistente de Investigación de Javier Torres Nafarrate (Proyecto Luhmann) y profesor de asignatura de la Universidad Nacional Autónoma de México.
} 


\section{I ntroducción}

El trabajo se divide en cuatro apartados que se refieren a a) una breve descripción del concepto de trastorno de la personalidad, b) el estado del arte de algunos estudios sobre la producción científica desde la sociología de la ciencia, en particular las propuestas de Karin Knorr-Cetina y la teoría del Actor-Red - que contempla el análisis de Bruno Latour, c) una articulación de la metodología de redes como plausible para el estudio del campo científico y d) una fundamentación dentro del marco conceptual de la teoría de los campos de Pierre Bourdieu asociada a la metodología de redes, donde se destaca que las variables utilizadas dentro de la producción científica son una forma de diferenciación interna de la ciencia.

\section{Historia de la locura o "enfermedad moral". Definición del trastorno de la personalidad}

El trastorno de la personalidad es una categoría analítica de la psiquiatría para el estudio de trastornos psiconeurológicos. En los años sesenta esta categoría emerge para el estudio clínico en dos esferas; por una parte, en la psiquiatría académica americana y en las prácticas psicoanalíticas, y por otro lado, en la historia de la locura o enfermedad moral (moral insanity) dentro de los estudios sobre la psicopatía. Sin embargo, la implementación del concepto en el tratamiento fundamentalmente psiquiátrico tiene registro esporádicamente en 1970 y se genera una implementación sistemática a partir de mediados los años noventa (Manning, 2002). En 1975 se reemplazó el término psicopatía por el de trastorno de la personalidad (Manning, 2002: 621). A pesar de que el trastorno de la personalidad y el término psicopatía no tienen consenso en tanto que uno sea evolución del otro, éstos son utilizados en distintas formas en Inglaterra, Estados Unidos y otros países de Europa (Manning, 2002). El tratamiento de dicho concepto dentro de la psiquiatría tiene un bajo grado de consenso, sobre lo cual se unifica un enunciado en el que el diagnóstico y la evaluación del trastorno de la personalidad son caóticos y que el diagnostico de la categoría en específico es pobre (Manning, 2002: 647).

Las clasificaciones psiquiátricas consisten en nomenclaturas y criterios de diagnósticos y existen dos medios que se encargan de dicha empresa. Por una parte, el documento que permite clasificar, diferenciar, describir y explicar el conocimiento de las enfermedades mentales es el Manual Diagnóstico y Estadístico de los trastornos mentales DSM-IV (Kaplan, Sadock, 2001:vii), que se presenta por primera vez en 1952. Por otro lado, los criterios de diagnóstico de los tratamientos son los que se expresan en la Clasificación de Estadística Internacional de las 
enfermedades y trastornos relacionados con la salud ( $\mathrm{ClE}-10)$ publicada por la Organización Mundial de la Salud. Un tratado firmado en el 2000 por los Estados Unidos y por la Organización Mundial de la Salud estableció que tanto lo tratado por el Manual Diagnóstico y Estadístico de los trastornos mentales como la Clasificación de Estadística Internacional de las enfermedades y trastornos relacionados con la salud, deben ser idénticos para asegurar la uniformidad en las estadísticas psiquiátricas nacionales e internacionales.

El Manual Diagnóstico y Estadístico (cuarta edición) define a los trastornos de la personalidad "como patrones permanentes de experiencia subjetiva y de comportamientos que se apartan de las expectativas de la cultura del sujeto" (Kaplan, Sadock, 2001:880). Dicho Manual estructura los trastornos en tres grupos: el grupo A contiene los trastornos de personalidad definidos como excéntricos, es decir, paranoide, esquizoide y esquizotípico; el grupo B comprende trastornos de la personalidad con una inclinación al dramatismo, a saber, antisocial, límite, histriónico y narcisista; por último el tercer grupo, grupo C, comprende personas que acostumbran ser temerosas y ansiosas lo que se conoce como trastorno de la personalidad por evitación, por dependencia y el obsesivo compulsivo. Además se agrega una categoría sobre el trastorno de la personalidad no especificado que comprende un trastorno pasivo-agresivo y depresivo.

Este concepto ha tenido un rápido desarrollo en los últimos veinte años dentro de las clasificaciones psiquiátricas, sin embargo, contiene numerosas controversias. A esto se suman interrogantes sobre los instrumentos que lo determinan, dónde comienza y dónde termina el trastorno de la personalidad y si los pacientes que lo padecen deben de ser hospitalizados o no. La evaluación del riesgo en torno a él es incierta y con ello genera una impopularidad hacia las políticas sociales empleadas y los tratamientos del personal médico. Así mismo, existe una cantidad de subcategorías muy amplia sobre el trastorno y es difícil poder deslindar una de otra. Los procedimientos de evaluación del riesgo según un grupo amplio de especialistas son inadecuados para identificar el peligro potencial de un individuo y en muchos de los casos existe una indeterminación en su detección.

Estos problemas sobre la evaluación, detección y tratamiento del trastorno de la personalidad se presentan al interior de la psiquiatría como especialidad. En ella se realizan producciones científicas expresadas en artículos que presentan dichas controversias. Con base en el prestigio de los investigadores, las revistas y las instituciones, el conocimiento expresado comunicativamente sirve como instrumento para la legitimación de una forma u otra de clasificación psiquiátrica 
que es social. Es justamente la lucha de legitimación y de interrelación entre investigadores y la ubicación de su posición en el mapa de producción en textos lo que surge como problemática que es objeto de análisis en este artículo.

\section{Breve estado de la cuestión en debate con Bourdieu: estudios sobre la producción científica desde la sociología de la ciencia}

En este apartado se pretende contextualizar la sociología de la ciencia propuesta por Bourdieu. Se presentan los principales debates del autor con sus contemporáneos y su ruptura con la etnometodología de la ciencia y la teoría del actor-red. Es precisamente dicha ruptura la que orienta su postulación sobre el campo científico, y con base en su propuesta enlazamos nuestro análisis, ya que una metodología de redes fundada en un análisis de coautorías hace visible la definición de campo como sistema de posiciones y disposiciones sociales que son objetivas y objetivantes.

El fundamento de las dos propuestas que presentaremos en adelante tiene un origen en la división y legitimación de lo que hasta hoy es la producción de conocimiento. La división se fundamentó en un análisis filosófico que hizo referencia a dos epistemologías; contexto de descubrimiento y contexto de justificación. Después de ciertas críticas al llamado positivismo lógico algunas investigaciones se orientaron a la ruptura de dicha distinción. Aquí analizaremos las propuestas de la teoría de Actor-Red y de Karin Knorr-Cetina.

Ante la división del contexto descubrimiento y contexto de justificación la sociología supuso que no existe nada fuera de lo social. Así, cuestionó las condiciones sociales del conocimiento, la verdad y la epistemología como algo relevante contrario a una tradición que se fundó en la ideología, las falsas creencias y prejuicios (Domenech, 1998: 16). Uno de los principales expositores de la teoría del Actor-Red es Bruno Latour. Latour formuló una propuesta sobre la economía política de la verdad. El fundamento de esta teoría supuso dos elementos; un principio de simetría y uno de heterogeneidad. De esta manera, la explicación y el contexto del conocimiento se fundaron en una distinción sumamente novedosa (y sumamente criticable) entre entidades humanas y no humanas que se relacionan mediante traducciones de relaciones de poder. La traducción de las relaciones de poder se basa en tres preguntas; cómo se definen los actores, cómo se asocian y cuáles son sus alianzas. Bourdieu hace un reconocimiento a Latour que debe tomarse con cierta advertencia ya que después presentaremos las críticas a esta propuesta, “Latour y Woolgar ponen en evidencia el importantísimo papel que, en el trabajo de fabricación de los hechos como ficción corresponde a los textos" (Bourdieu, 2001: 53). Siguiendo a 
Bourdieu haremos críticas a esta teoría. El análisis sobre el trabajo de Pasteur realizado por Bruno Latour, según Bourdieu, toma a Pasteur como significado textual dentro de una red heterogénea de entidades donde lo "semiológico se combina con una visión ingenuamente maquiavélica de las estrategias de los científicos" (Bourdieu, 2001: 56). Agregando argumentos a lo escrito por Bourdieu, la tesis de la simetría resulta un planteamiento problemático, ya que cómo se le da el mismo peso a la existencia de cosas no materiales y las prácticas humanas en el proceso de construcción de conocimiento. Existen evidentemente críticas mucho más acabadas a esta propuesta que no podremos agotar aquí, pero vale la pena mostrar los problemas que contempla ahora que ha sumado tantos seguidores.

La propuesta de Karin Knorr-Cetina se basa en dos fundamentos; la etnometodología y el constructivismo radical. Knorr-Cetina cita a Pierce sobre el proceso de investigación científica y refiere que "el proceso de investigación científica (su contexto de descubrimiento), omitido por el objetivismo, es en sí mismo el sistema de referencia que hace posible la objetivación de la realidad" (Knorr Cetina, 2005: 54), lo cual es una evidencia de la propuesta de la universalidad social del conocimiento. Para Knorr-Cetina la investigación es un proceso de fabricación. El dato es una construcción del investigador en un laboratorio y no refleja la preocupación científica cuasi omnipotente de la verdad, sino a los intereses de un razonador práctico en la unidad de investigación que es el laboratorio. Propone el interaccionismo metodológico, el cual se enfoca principalmente en la práctica, en la "interacción como la forma más adecuada de explicación, forma de la cual emergen las características contextuales y temporales de la acción" (Knorr Cetina, 2005: 94). Es decir, cómo los científicos producen y reproducen su conocimiento en el laboratorio.

La razón por la cual tomamos el marco teórico de los campos de Bourdieu está apoyado en una crítica a lo anteriormente presentado. El problema de Knorr-Cetina (según Bourdieu) reside justamente en basarse en una visión interaccionista que ignora el principio de una estructura y de las disposiciones que suponen el marco de acción y de interacciones. Cuando se estudia la práctica en el laboratorio y en la creencia que tienen los investigadores "no se interroga en absoluto acerca de las condiciones estructurales de la producción de la creencia" (Bourdieu, 2001: 44). La propuesta de Bourdieu es la noción de campo ya que para él dicho concepto es el principio de las acciones que están en las posiciones y en las disposiciones de la estructura de poder. 
Antes del siguiente apartado hay que dejar dos argumentos claros. Por un lado, en la teoría de los campos es relevante la comunicación científica ya que ésta expresa una relación estructural entre un grupo de personas que comparten un background de problemas y presupuestos. Se construyen textos donde el mundo físico parece hablar y actuar por sí mismo. Por otro lado, la producción de textos científicos es relevante para la sociología de la ciencia pues "sin escritura la ciencia no podría seguir existiendo" (Knorr Cetina, 2005: 82). Ya que cuando se trata de producción científica ninguna teoría podría pensar que el análisis de textos es irrelevante, sólo pensemos la importancia de Nature o de ISI Web of Science en la forma de relacionarse entre investigadores. La ciencia también se describe por sus textos, en otras palabras los textos son descripciones de la ciencia. (Luhmann, 1996: 60).

\section{Metodología de Redes en un marco del campo científico}

En los siguientes apartados metodológicos introduciremos algunas descripciones del marco de mediciones de la sociología de la ciencia en el cual este trabajo se enmarca. Después se mostrarán los tres indicadores que se han retomado para el análisis.

\section{Cienciometría}

La definición de los análisis cienciométricos se podrían explicar como una referencia a un principio de reflexividad científico. La ciencia hace ciencia tomándose a sí misma como objeto y buscando identificar sus propias leyes y generalidades que rigen la actividad científica en su globalidad (Callon, et al 1993). No sólo es el objeto de investigación sino que se aplica sus propias metodologías. Según MacíasChapula (1998), la cienciometría es el estudio de los aspectos cuantitativos de la ciencia como disciplina y actividad económica. Sus áreas de investigación se concentran en estudios estadísticos del lenguaje, frecuencia de citaciones, características de las publicaciones, la relación autor-productividad, medida por el número de artículos o por el grado de colaboración. O bien, por el análisis de citas, distribución de autores, revistas, instituciones o países (Macías- Chapula, 1998). Algunas de las primeras referencias de estos estudios se realizaron mediante análisis de citación en Estados Unidos por Garfield y De Solla Price (Callon, et al 1993). Sin embargo, el antecedente más importante que permite mediciones cuantitativas de la ciencia es la creación en 1963 de Science Citation Index (SCI).

Además de lo mencionado por Macías-Chapula, los estudios cienciométricos establecen relaciones entre investigación e innovación pues pasan necesariamente por el análisis sistemático de las producciones literarias de los investigadores e 
ingenieros (Callon, et al 1993). Así, según Callon, Courtial y Penan, la producción de conocimientos certificados es la más grande empresa literaria (Callon, et al 1993).

\section{Redes de coautoría}

Según Moody "scientific collaboration networks affect scientific practice" (Moody, 2004: 213). Las redes de coautoría entre científicos como práctica establece redes de individuos a los cuales les interesa "maximizar su(s) agujeros estructurales, es decir, su capacidad de conectar o acceder a nodos no redundantes" (Molina, Muñoz, Domenech, 2002: 5). El análisis de colaboraciones permite identificar y representar redes entre instituciones, países e investigadores. Algunos resultados que los estudios de redes de coautorías entre países, instituciones e investigadores han mostrado son que la probabilidad en la colaboración entre autores difiere según áreas espaciales y áreas temáticas. Por ejemplo, el estudio de Moody sobre la estructura de las redes de colaboración en las ciencias sociales refiere que los análisis cuantitativos tienen mayor probabilidad de ser trabajados por diferentes autores que los cualitativos (Moody, 2004). Del mismo modo, la discusión sobre las publicaciones en América Latina y el Caribe hace énfasis en la diferencia en formas de colaboración científica entre estos dos tipos de acercamientos metodológicos (Collazo-Reyes, et al 2008).

\section{Análisis de citas}

El estudio sobre el prestigio y la relación entre investigadores se ha fundamentado en un análisis de citas. Así, "el medio más común para otorgar méritos y reconocimiento en la ciencia es mediante las citas" (Macías- Chapula, 1998). Al menos pueden reconocerse dos mecanismos de estratificación científico donde el análisis de citación resulta de suma utilidad. Por una parte, los estudios sobre el idioma de las publicaciones muestran que el número de citaciones que un artículo recibe puede depender del lenguaje en el cual es publicado (Collazo-Reyes, et al 2008). De este modo, la probabilidad de ser citado aumenta si la publicación se hace en inglés. Los científicos buscan la visibilidad internacional al publicar en "mainstream journals" (Collazo-Reyes, et al 2008) que son escritas en dicho idioma. En una región como México, y en América Latina en general, los estudios realizados por Garfield (1984) mostraron una reducida inter-citación, de tal forma se trazó una distinción entre la producción local, regional y externa de artículos.

Sentadas las bases de los estudios cienciométricos es pertinente establecer qué puntos son retomados por nuestro análisis para conjugar un caso empírico de 
estudio, un análisis metodológico con herramientas de los estudios de redes y cienciométricos y una aplicación teórica desde la propuesta de los campos de Pierre Bourdieu.

Para la recolección de los datos en la base de datos Scopus (caso empírico de estudio), el día 21 de Junio del 2010 se realizó una búsqueda con los descriptores: TITLE-ABS-KEY("PERSONALITY DISORDER") AND AFFIL (mexico) AND NOT AFFIL (new mexico) AND PUBYEAR AFT 1969. Dicha búsqueda arrojo un total de 72 artículos que se contemplan como la muestra de nuestro estudio.

Así, en el análisis se utilizan tres categorías para la investigación de la producción científica realizada por investigadores mexicanos sobre el trastorno de la personalidad; citación, productividad y productividad con referencia a países. Estos indicadores nos muestran, por un lado el número de trabajos como reflejo de la producción científica, el número de citas como el impacto de los artículos y la coautoría como representación del grado de colaboración en la ciencia en niveles nacionales e internacionales (Macías- Chapula, 1998). Estos mapas de científicos y países ubican su posiciones relativas a la colaboración científica mundial.

En el estudio de Molina, Muñoz y Domenech se reconocen al menos tres formas de análisis de coautorías; egocéntrica, bola de nieve y listar autores en un campo de investigación (Molina, Muñoz y Domenech, 2002). Nuestro estudio resulta cercano a este último análisis, sin embargo, habría que enfatizar que lo relevante no es sólo cómo es que los investigadores se relacionan, sino cómo es que se relacionan en un campo como estructura de relaciones frente a un tema, más que sólo mostrar los casos particulares e interacciones entre autores.

Para analizar la citación, productividad y productividad con referencia a países se utilizará la centralidad de grado. "The degree of a vertex is the number of lines incident with it" (Denooy, 2000: 63). Para observar la citación se propone analizar el número de citas que recibe un artículo, es decir, la centralidad de grado de entrada. "The indegree of a vertex is the number of arcs it receives" (Denooy, 2005: 64). Las relaciones de entrada definen al artículo más citado como el más prominente.

La productividad se refiere al número de artículos que produce un autor. Este número de artículos se considera como un atributo del nodo que al ser contrastado con la centralidad de grado, es decir, el número de citaciones expresa la posición que ocupa un autor con respecto a otros como posición de poder (prestigio). El análisis relacional entre la productividad científica y los patrones de citación ha sido ampliamente trabajado por Derek De Solla Price (1973). La tesis central de este es 
que la importancia de una teoría cientifica está expresada por el prestigio de un autor en tanto su volumen de productividad y su nivel de citación (Vélez, 2010: 68). Al hacer una conjugación de la teoría de los campos de Bourdieu y lo expresado por De Solla Price se puede referir que el prestigio opera como un polo de atracción para otros investigadores y científicos, puesto que muestra las interacciones de los investigadores como relaciones de poder. Así mismo, una categoría que ha sido trabajada tanto por Bourdieu como por la teoría de redes es la categoría de capital social. El capital social2 puede ser utilizado para movilizar ciertos recursos tanto materiales como simbólicos, cuanto mejor posición tenga un investigador en el campo, mayor movilidad le ofrece su conocimiento al interior.

A los índices anteriores se les agrega un atributo más a los nodos, la productividad distribuida geográficamente. Esto permite observar la distribución del prestigio en el campo científico mexicano de los investigadores que escriben sobre el trastorno de la personalidad con respecto a otros países.

\section{Características del campo}

De las críticas a la teoría del Actor-Red y Knorr-Cetina, Bourdieu describe:

"Uno de los puntos centrales por los que me distancio de todos los analistas que acabo de recordar es el concepto de campo, que pone el acento sobre las estructuras que orientan las practicas científicas y cuya eficacia se ejerce a una escala macrosociológica en la que se sitúan la mayoría de los trabajos que he criticado y, en especial, los estudios de laboratorio" (Bourdieu, 2001: 63).

Resulta insuficiente el análisis interaccionista de la sociología de la ciencia presentado en uno de los anteriores apartados, ya que será necesario develar un código interno desde donde se realizan dichas interacciones. Por un lado, el código es la estructura de relaciones objetivas entre los laboratorios, instituciones, universidades, etc. Por el otro, opera en los investigadores que dirigen y orientan sus prácticas dentro de la ciencia como disposiciones de la misma estructura. El campo científico es un campo de fuerzas dotado de una estructura y de agentes que la soportan.

Son dos los conceptos que aparecen íntimamente ligados en la teoría de Bourdieu desde la estructura; espacio social y campo. El primero tiene que ver con las estructuras de las relaciones objetivas en general de la sociedad, "el espacio social en tanto que espacio objetivo, estructura de relaciones objetivas que determina la forma que pueden tomar las interacciones y la representación que de ellas pueden

\footnotetext{
${ }^{2}$ En el análisis de redes existe una basta discusión sobre la definición sobre el concepto capital social, aquí es retomado desde la teoría de Bourdieu, con la importancia que tiene este capital como el económico, el cultural y el simbólico.
} 
tener aquellos que se encuentran en dicho espacio o estructura." (Bourdieu, 2003a: 241). El campo es una especie de tablero de ajedrez que tiene determinadas ciertas posiciones que sólo específicas figuras pueden ocupar, unas reglas del juego, un código que regula cómo se juega y una illusio que según Bourdieu opera como creencia en el juego y lo que está en juego (Bourdieu, 2003b: 93). "Solamente en el campo de posiciones se definen tanto los intereses genéricos asociados al hecho de participar en el juego como los intereses específicos ligados a las diferentes posiciones $\mathrm{y}$, a través de ellos, la forma y el contenido de las posturas en las que se expresan estos intereses." (Bourdieu, 2003a: 10).

Entonces, el campo es un microcosmos dentro del macrocosmos que constituye el espacio social global (Lahire, 2002: 2), contiene las reglas del juego y estrategias de intereses que no se reducen a lo que sucede sólo en el campo. Supone además una estructura de posiciones que expresa las luchas entre los agentes para la ocupación de una posición, donde lo que opera son capitales desiguales entre ellos. Todo agente se relaciona con esas posiciones mediante intereses que incorpora a un sistema de disposiciones llamado habitus.

\section{La producción científica mexicana sobre el trastorno de personalidad; la disciplina psiquiátrica en el campo científico}

El campo en el que se introduce nuestro análisis es el campo científico mexicano, el cual se limita a la producción sobre el trastorno de la personalidad para la psiquiatría. Al vincular las redes como una metodología para el estudio del campo, en el análisis de la productividad (centralidad de grado de entrada), la productividad distribuida geográficamente y la citación (centralidad de grado) puede ubicarse la posición ocupada por los investigadores en torno al prestigio, que es una forma de capital social y simbólico. Los investigadores están unidos por luchas y alianzas ligadas con la posición que ocupan en dichas luchas. El investigador consume por medio de la citación pues es un agente que reproduce el campo y toma posición con respecto a las citas, por ejemplo, en México el Sistema Nacional de Investigadores toma la publicación y la citación como un referente de productividad. 


\section{Numero de citas por artículo}

\begin{tabular}{|c|c|c|c|c|c|}
\hline NR & Autores & $\mathrm{TI}$ & PY & VL & TC \\
\hline $\mathrm{n} 1$ & $\begin{array}{l}\text { Jimenez, F } \\
\text { Velasco, F } \\
\text { Salin-Pascual, R } \\
\text { Hernandez, JA } \\
\text { Velasco, M } \\
\text { Criales, J L } \\
\text { Nicolini, H }\end{array}$ & $\begin{array}{l}\text { A patient with a resistant major } \\
\text { depression disorder treated with deep } \\
\text { brain stimulation in the inferior } \\
\text { thalamic peduncle }\end{array}$ & 2005 & 57 & 56 \\
\hline $\mathrm{n} 2$ & $\begin{array}{l}\text { Brailowsky, S } \\
\text { Garcia, O }\end{array}$ & Ethanol, GABA and epilepsy & 1999 & 30 & 30 \\
\hline n3 & $\begin{array}{l}\text { Johnson, E } \\
\text { Arria, AM } \\
\text { Borges, G } \\
\text { Ialongo, N } \\
\text { Anthony, JC }\end{array}$ & $\begin{array}{l}\text { The growth of conduct problem } \\
\text { behaviors from middle childhood to } \\
\text { early adolescence: Sex differences } \\
\text { and the suspected influence of early } \\
\text { alcohol use }\end{array}$ & 1995 & 56 & 30 \\
\hline $\mathrm{n} 4$ & Frias-Armenta, M & $\begin{array}{c}\text { Long-term effects of child punishment } \\
\text { on Mexican women: A structural } \\
\text { model }\end{array}$ & 2002 & 26 & 14 \\
\hline n5 & $\begin{array}{c}\text { Saez-De-Ocariz, M } \\
\text { Orozco-Covarrubias, L } \\
\text { Mora-Magana, I } \\
\text { Duran-McKinster, C } \\
\text { Tamayo-Sanchez, L } \\
\text { Gutiérrez-Castrellon, P } \\
\text { Ruiz-Maldonado, R }\end{array}$ & $\begin{array}{c}\text { Dermatitis artefact in pediatric } \\
\text { patients: Experience at the national } \\
\text { institute of pediatrics }\end{array}$ & 2004 & 21 & 13 \\
\hline n6 & $\begin{array}{c}\text { Borges, G } \\
\text { Cherpitel, CJ } \\
\text { Medina-Mora, ME } \\
\text { Mondragon, L }\end{array}$ & $\begin{array}{l}\text { Violence related injuries in the } \\
\text { emergency room: Alcohol, depression, } \\
\text { and conduct problems }\end{array}$ & 2004 & 39 & 12 \\
\hline $\mathrm{n} 7$ & $\begin{array}{c}\text { Archer-Dubon, C } \\
\text { Orozco-Topete, R } \\
\text { Reyes-Gutierrez, E }\end{array}$ & Two cases of psychogenic purpura & 1998 & 50 & 12 \\
\hline n8 & $\begin{array}{c}\text { Apiquian, R } \\
\text { Paez, F } \\
\text { Loyzaga, C } \\
\text { Cruz, E } \\
\text { Gutierrez, D } \\
\text { Suarez, JA } \\
\text { Ulloa, RE } \\
\text { Fresan, A } \\
\text { Vallejo, G } \\
\text { Nicolini, H } \\
\text { Medina-Mora, ME }\end{array}$ & $\begin{array}{c}\text { Mexican study on the first psychotic } \\
\text { episode: Preliminary results, } \\
\text { sociodemographic and clinical } \\
\text { charateristics [Estudio Mexicano sobre } \\
\text { el primer episodio psicótico: } \\
\text { Resultados preliminares, } \\
\text { características sociodemográficas y } \\
\text { clínicas] }\end{array}$ & 1997 & 20 & 9 \\
\hline n9 & $\begin{array}{c}\text { Del Mar Sáez De Ocariz, } \\
\text { M } \\
\text { Nader, JA } \\
\text { Del Brutto, OH } \\
\text { Santos Zambrano, JA }\end{array}$ & $\begin{array}{c}\text { Cerebrovascular complications of } \\
\text { neurosyphilis: The return of an old } \\
\text { problem }\end{array}$ & 1996 & 6 & 9 \\
\hline $\mathrm{n} 10$ & $\begin{array}{c}\text { Lara, C } \\
\text { Fayyad, J } \\
\text { de Graaf, R } \\
\text { Kessler, RC } \\
\text { Aguilar-Gaxiola, S } \\
\text { Angermeyer, M } \\
\text { Demytteneare, K } \\
\text { de Girolamo, G } \\
\text { Haro, J M } \\
\text { Jin, R } \\
\text { Karam, EG } \\
\text { Lepine, J-P } \\
\text { Mora, MEM } \\
\text { Ormel, J } \\
\text { Posada-Villa, J } \\
\text { Sampson, N }\end{array}$ & $\begin{array}{l}\text { Childhood Predictors of Adult } \\
\text { Attention-Deficit/Hyperactivity } \\
\text { Disorder: Results from the World } \\
\text { Health Organization World Mental } \\
\text { Health Survey Initiative }\end{array}$ & 2009 & 65 & 7 \\
\hline
\end{tabular}




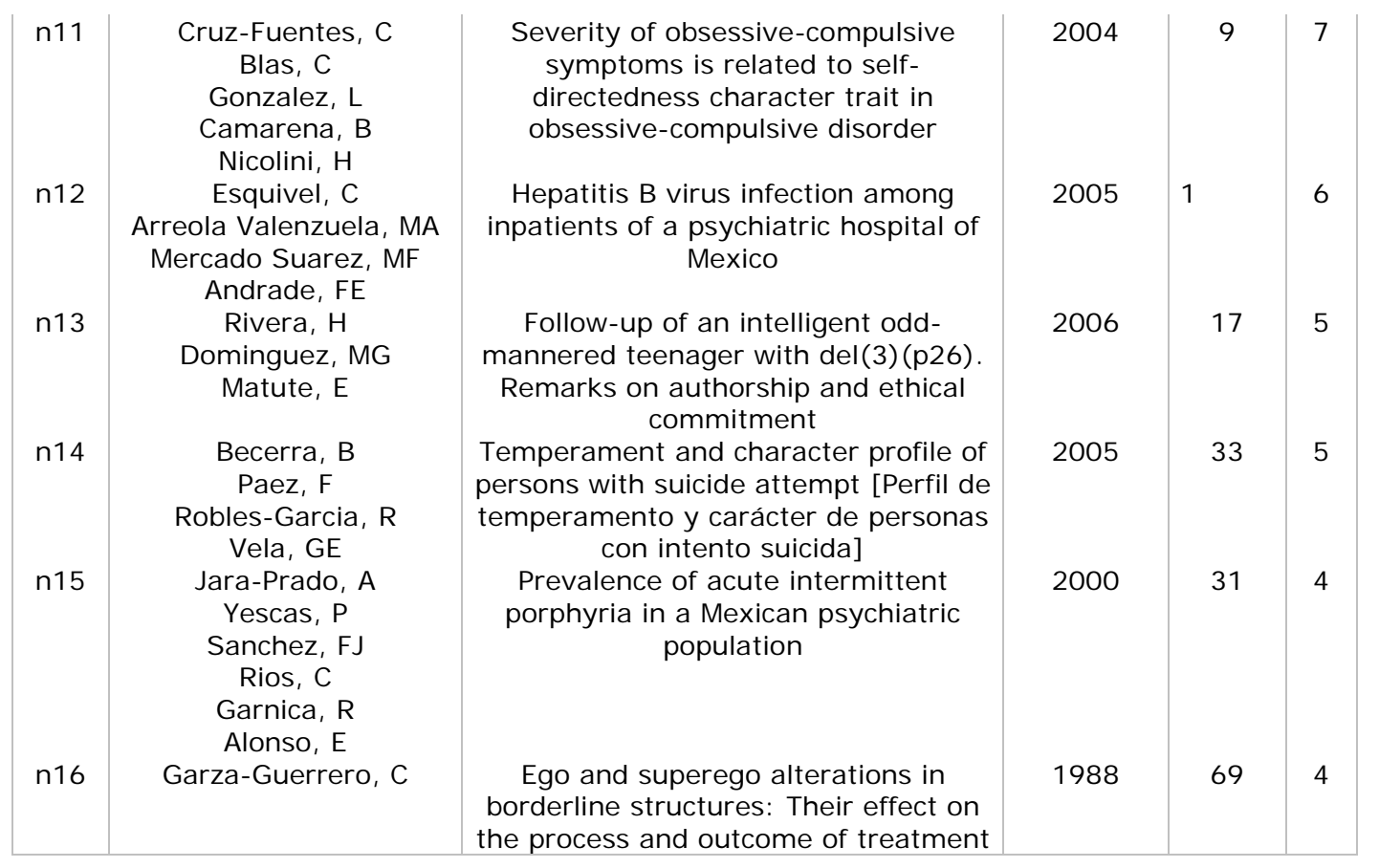

Tabla 1. Número de citas por artículo.

En la tabla $1^{3}$ se presenta la relación de los artículos por el número de citas que tienen de acuerdo con lo que aparece en la base de datos Scopus. En el primer artículo (n1) se presenta una coautoría entre siete investigadores que trabajan en Instituciones mexicanas. La afiliación de los autores está relacionada a unidades de radiocirugía y nerocirugía del Hospital General de México, el Departamento de Psiquiatría de Instituto Nacional de Ciencias Médicas y Nutrición y el Departamento de Ciencias Genómicas de la Universidad de la Ciudad de México. El nombre del artículo es "A patient with a resistant depresión disorder treated with deep brain stimulation in the inferior thalamic peduncle" y es publicado en la revista Neurosurgery en el 2005.

El segundo artículo (n2) fue escrito por dos autores que muestran una afiliación al Instituto de Fisiología Celular de la Universidad Nacional Autónoma de México. (UNAM). El nombre del artículo es "Ethanol, GABA and epilepsy" y se publicó en inglés en 1999 en la revista Archives of Medical Research. Esta revista ahora publica en inglés aunque originariamente lo hacia en español, al cambiar el lenguaje atrajo el interés internacional, sin embargo, mas del 50\% de los artículos y de la citación recibida es de la comunidad científica mexicana (Collazo-Reyes, et al, 2008).

\footnotetext{
${ }^{3}$ La función de la tabla es presentar artículos que tienen cuatro o más citas (ver anexo) ya que se pretende enfocar en el prestigo vía la citación y no las relaciones totales entre los artículos. El número total de artículos es 72 de los cuales 22 tienen una sola cita y 21 no tienen ninguna.
} 
El tercer artículo (n3) es una coautoría entre cinco investigadores de afiliación al Instituto Mexicano de Psiquiatría hoy Intituto Nacional de Psiquiatría. El nombre del artículo es "The growth of conduct problem behaviors from middle childhood to early adolescence: Sex differences and the suspected influence of early alcohol use" y se publicó en Journal of Studies on Alcohol.

El cuarto artículo (n4) fue escrito por un investigador del Departamento de Leyes de la Universidad de Sonora en México. El nombre del artículo es "Long-term effects of child punishment on Mexican women: A structural mode" publicado en Child abuse and Neglect.

El quinto artículo (n5) fue escrito por siete investigadores pertenecientes al Instituto Nacional de Pediatría. El nombre del artículo es "Dermatitis artefact in pediatric patients: Experience at the national institute of pediatrics" publicado en la revista Pedratric Dermatology.

Sobre esto se puede hacer una primera conclusión, y es que, según la base de datos de Scopus, en el análisis de las citaciones de artículos, los niveles más altos de citaciones son derivados de artículos realizados por investigadores laborando en instituciones mexicanas.

Lo anterior se hace relevante ya que existen coautorías con universidades e instituciones extranjeras que no tienen un nivel alto de impacto con referencia a la citación. Además el Ranking Web of Word Hospitals ${ }^{4}$ contempla en su lista al Instituto Nacional de Psiquiatría a nivel continental en el número 48 y mundial es 1 , 838. Dicho Ranking no esta divido por disciplinas, es decir, contempla hospitales en general. Y según el Ranking Web de Universidades del mundo ${ }^{5}$ la UNAM se encuentra en el número dos en América Latina y 44 a nivel mundial. Aparece una relación directa entre el prestigio de los investigadores por el número de citas y de las Instituciones desde donde publican. Bajo el marco del análisis del campo se puede leer que la base de la estructura del prestigio de las Universidades y las Instituciones se expresa en el prestigio de los investigadores y de modo inverso, el prestigio de los investigadores contribuye al prestigio de las Instituciones. Así el campo por sus disposiciones se retroalimenta en una relación dialéctica.

Sobre el campo científico, Bourdieu distingue dos tipos de capital, el de autoridad y el del poder sobre el mundo científico. Este análisis se acerca más al primero que al segundo. Además se destaca una nueva dimensión del análisis que parte de la

\footnotetext{
${ }^{4}$ Tomado de http://hospitals. webometrics.info/.

${ }^{5}$ Tomado de http://www. webometrics.info/top100_continent_es.asp?cont=latin_america. Sin embargo, existe una disputa interna a los rankings ya que la UNAM aparece en otros como la más importante.
} 
productividad y la citación. Sin embargo, la medición del prestigio resulta insuficiente sólo desde un análisis de las dos categorías anteriores, las formas de diferenciación científica se realizan por la autoridad ejercida mediante el prestigio de la afiliación (la universidad desde donde se publica). Así, como resultado de la difereciación interna de la ciencia y de la imposición que aparece como legítima (aunque debería de describirse como una arbitrariedad cultural), el campo científico como estructura de posiciones objetivas contiene una censura legítima de los dispositivos de admisión. ¿Quiénes son los más citados?, ¿dónde publican? ¿en qué idioma?

La valoración de la obra científica está supeditada por la posición ocupada por los investigadores orientada por las anteriores preguntas. Lo que se produce y busca publicar es una estrategia social de inversión orientada al beneficio específico del capital social y científico. ${ }^{6}$

\section{Productividad de los investigadores}

Según Bourdieu "el premio al crédito científico no es el dinero sino las recompensas garantizadas por la valoración de los colegas" (Bourdieu, 2003b: 96). En el campo científico un autor es un miembro que hace representaciones oficiales mediante las citas. Las citas le dan una visibilidad social (Bourdieu, 2003b: 92) y reconocimiento en las relaciones de comunicación.

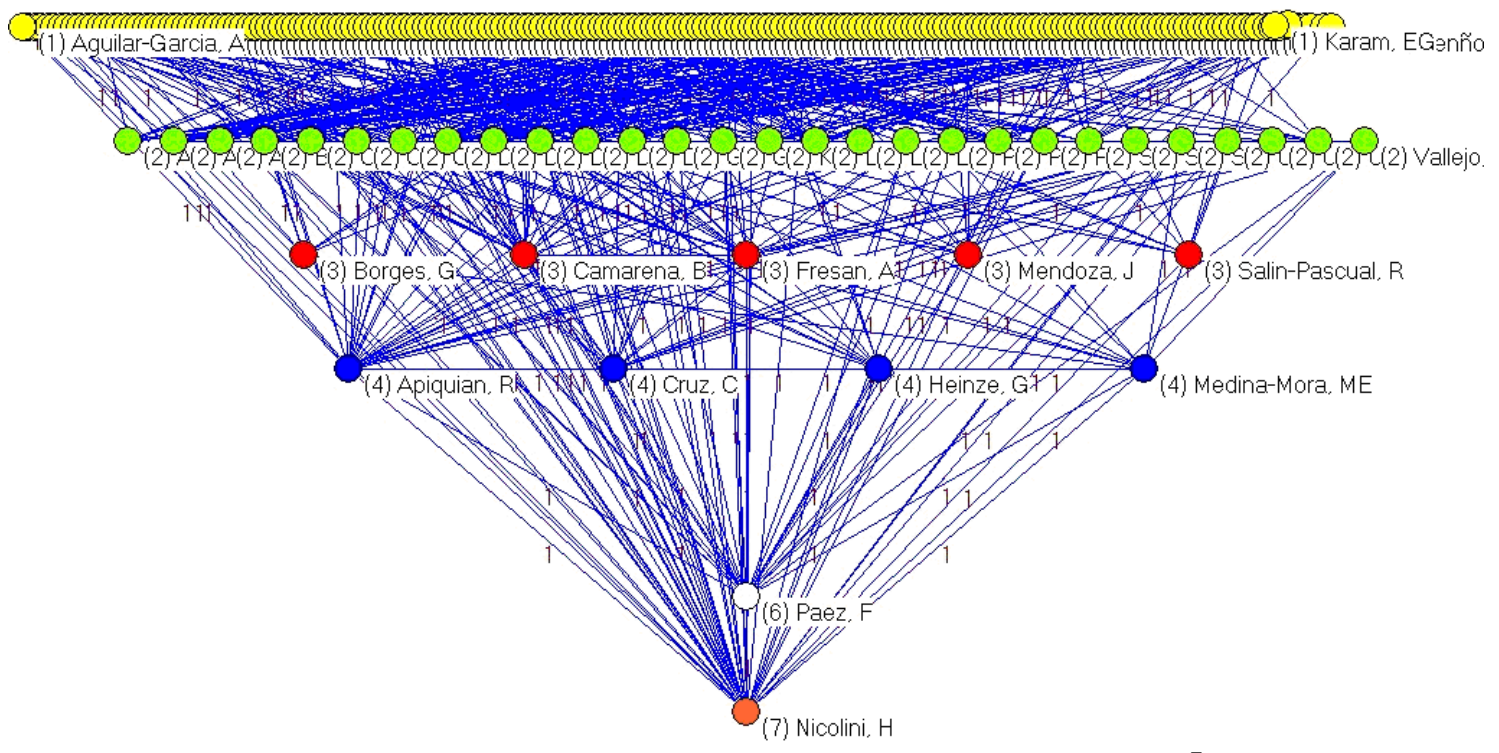

Figura 1. Productividad de los investigadores de mayor a menor ${ }^{7}$.

\footnotetext{
${ }^{6}$ A lo anterior habría que hacer una nota. Bourdieu distingue dos áreas de la ciencia; la competencia científica y competencia social. Es evidente que nuestro análisis se encuentra en la primera, en mostrar la estructura del campo por el prestigio de las citaciones, de las intituciones que publican, donde se publica y las coautorías. Otro análisis sería ver las consecuencias de dichas publicaciones al nivel social.

7 Nota: La gráfica 1 muestra la centralidad de grado de entrada expresada como productividad. Se encuentra organizada desde el grado mayor de productividad en la parte de abajo y los autores con
} 
Según esta red, el autor que tiene más publicaciones en las bases de Scopus sobre trastorno de la personalidad es $\mathrm{H}$ Nicolini con siete artículos. La información que arroja la base de datos tomando en cuenta la afiliación nos muestra que $\mathrm{H}$ Nicolini es un investigador que ha trabajado (o se encuentra trabajando) en el Departamento de Genética Psiquiatrica del Instituto Mexicano de Psiquiatría, el Departamento de Ciencias Genómicas de la Universidad Autónoma de la Ciudad de México, el Servicio de Psiquiatría del Hospital Español, la división de Investigaciones Clínicas del Instituto Mexicano de Psiquiatría, en el Departamento de Psiquiatría y de Salud Mental de la Universidad Nacional Autónoma de México y en el Laboratorio de Genética de la Subdirección de Investigaciones Clínicas.

El autor tiene un conocimiento amplio del campo científico mexicano en materia de salud mental y de investigación sobre el trastorno de la personalidad. Además Nicolini es uno de los seis autores que escriben el artículo más citado (N1 con 56 citas). Así, la tesis sobre la estrecha relación entre la productividad y el grado de citaciones se comprueba en un principio con este autor, los que más producen son los autores con el mayor número de citas. Si se le agrega la nueva dimensión en nuestro análisis, nos permite afirmar que son también los que logran un mayor posicionamiento dentro del campo. El posicionamiento de este autor es de gran relevancia, pues ha desempeñado labores en diversas divisiones del hospital y la universidad más prestigiosos en México según el Ranking mundial.

El segundo autor que aparece en la gráfica 1 con un grado mayor en el número de publicaciones es Páez F. Este segundo autor al igual que Nicolini ha trabajado en las instituciones que hemos resaltado como las más prestigiosas, en la División de Investigaciones Clínicas del Instituto Mexicano de Psiquiatría, el Departamento de Psiquiatría y Salud Mental de la Universidad Nacional Autónoma de México y en el Departamento de Investigación de la Subdirección de Calidad y Desarrollo I en Jalisco. A diferencia del primer autor, Páez no aparece en el artículo más citado, pero si en el artículo "Estudio Mexicano sobre el primer episodio psicotico: Resultados preliminares, características sociodemográficas y clínicas" (n8) que tiene 9 citas. Su productividad nos muestra que el prestigio de las instituciones donde laboran lo posiciona favorablemente al interior del campo.

menor grado de productividad en la parte superior. Así, el nodo color naranja es H. Nicolini y la línea 7 es el número de citas, las líneas son justamente las que organizan de menor a mayor la tabla. El segundo vértice de color blanco lo ocupa $\mathrm{F}$. Paez con 6 números de líneas. Así, para leer la tabla se parte que los colores distinguen a los autores por el número de artículos que producen, número que aparece en las líneas (relaciones). 
Puede decirse entonces que el factor afiliación a una institución determina el posicionamiento de los autores, tanto como la productividad y la citación. La relación entre agentes e instituciones son mediadas por relaciones de poder y prestigio. Sin embargo el poder no habrá de entenderse sólo como una imposición, sino como posibilidad de movilidad dentro de un campo. Ya Foucault había referido que las relaciones de verdad como las de la ciencia son relaciones de poder "que se realizan especialmente a través de las relaciones de conocimiento y de comunicación" (Bourdieu, 2003b: 100). Los investigadores como agentes dentro de un campo operan capitales, en este caso el capital social y el capital científico, los cuales les generan una suerte de competencia y de pertenencia al interior del campo.

El juego del campo científico se encuentra en la legitimidad de los autores que tienen acceso al campo, lo cual genera una forma especial de censura. Entonces, ¿quién está dentro del campo y quién no? En palabras de Bourdieu, esto depende de quién tiene el monopolio legítimo de la representación científicamente legítima de lo real (Bourdieu, 2003b: 123).

\section{Productividad con referencia a países}

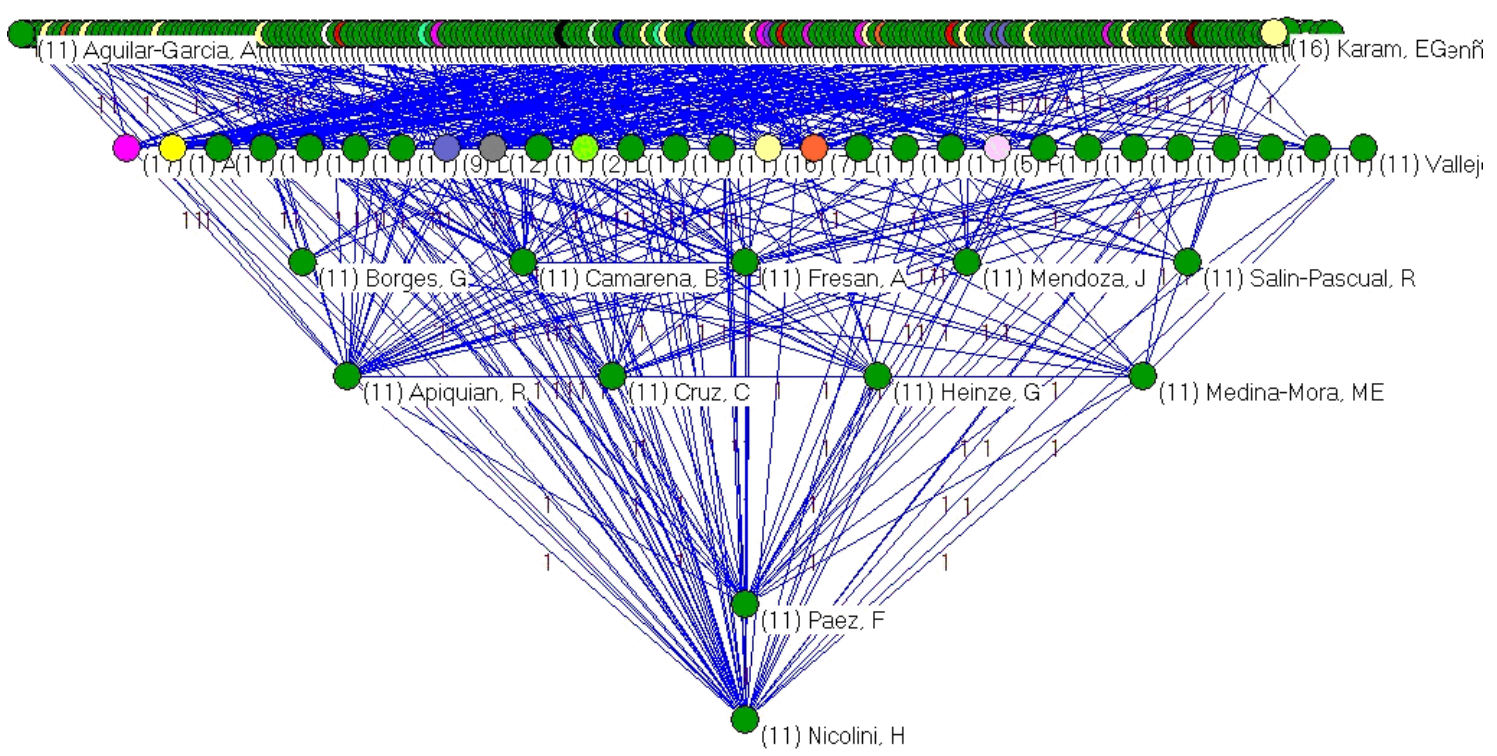

Figura 2. Productividad de los investigadores de mayor a menor por países ${ }^{8}$.

Lo primero que se desprende de la gráfica es que el grado más alto de coautorías es entre mexicanos. Siguiendo el análisis, el primer punto que podemos reconocer de un país distinto a México es J, Lapine. Lapine tiene una afiliación al Hospital de

\footnotetext{
${ }^{8}$ La gráfica 2 muestra que el color verde y el número de relaciones 11 son mexicanos, el color naranja refiere al investigador de Francia con el número de relaciónes 7. Degraaf con el color gris es holandés con un número de relaciónes 12 y Angermeyer es amarillo con un número de relaciónes 1 .
} 
Asistencia Pública de París y al Departamento de Psiquiatría del Hospital Fernand Widal. No obstante que el autor contempla el mayor número de coautorías con autores de afiliación mexicana, los artículos en los que escribe no aparecen en la tabla de los más citados.

En la misma posición de la red aparecen tres autores; Angemeyer M, De Graaf R y Kessler RC. Angemeyer ha laborado en el Centro de Salud Mental Público de Austria. De Graaf R presenta una afiliación al Departamento de Epidemiología del Instituto de Salud Mental y Adicciones de Holanda. Kressler RC es parte del Departamento de Políticas del Cuidado de la Salud de la Escuela de Medicina de Harvard en Boston, Estados Unidos.

Aunque no es el tema de este artículo, con relación a Latinoamerica, Posada-Villa es el investigador que más artículos escribe con investigadores mexicanos, este está afiliado al Colegio Mayor de la Universidad de Cundinamarca y al Ministerio de Protección Social en Colombia.

\section{Conclusiones}

Para Bourdieu el sujeto de la ciencia es "el campo científico en cuanto universo de relaciones de comunicación y de concurrencia reguladas en materia de argumentación y de verificación" (Bourdieu, 2003b: 125). El presente trabajo muestra una relación endógena entre mexicanos, es decir, los mexicanos citan mexicanos en la producción sobre el trastorno de la personalidad.

Al relacionar la productividad con la citación se muestra que el investigador que ha escrito más artículos es coautor del artículo más citado. Sumado a esto se puede referir que el prestigio por la universidad o al instituto al cual se pertence, se expresa por el prestigio de la productividad y del conocimiento de la lógica interna al campo. Es decir, los autores que escriben en el artículo con mayor número de citas muestran su conocimiento sobre la lógica interna, puesto que el conocimiento de las posiciones les permite la movilidad dentro de la lógica de admisión y competencia científica.

Se muestra una tendencia entre las relaciones de las categorías tratadas; citación, productividad, productividad por países e institución donde se labora sobre el prestigio de los autores que publican sobre el transtorno de la personalidad en México. Esta tendencia puede ser expresada como una suerte de determinación de las categorías sobre el prestigio, como momentos coercitivos de las relaciones de la estructura de un campo y sus agentes como operadores. 
Partiendo de nuestras conclusiones podemos referir al menos a tres puntos; a) existe una cierta orientación sobre el tema por prestigio interno (endógeno), es decir, por lo que en México se produce y no por la relación con las universidades extranjeras, b) la tesis de De Solla Price sobre el prestigio como polo atractor y resultado del análisis entre la citación y los investigadores con mayor productividad se cumple al menos en el caso de Nicolini, y, c) que las instituciones y los investigadores presentan una relación directa por el prestigio. Así mismo podemos afirmar que el capital simbólico y científico se encuentra estrechamente ligado a los procesos de legitimación por el conocimiento y lo que se comunica de este.

\section{Apuntes finales}

Este análisis propuso trazar las primeras líneas sobre la investigación de la producción científica mexicana en torno al trastorno de la personalidad. Sin embargo existen ciertos problemas que se pueden mostrar siguiendo el esquema de la teoría de campos y el análisis de la citación y la productividad.

La reflexividad sociológica propuesta por Bourdieu hace la recomendación de objetivar al sujeto objetivante, es decir, hacer visible el modo de operar sociológico. Este operar debe referir a los problemas internos de la teoría.

Por una parte explica Latour que "analyses of citations in specific papers tend to tell us little about the substance of the papers; macroanalyses of science funding remain virtually silent on the nature of intellectual activity" (Latour, 1986: 18) Siguiendo esto, según Bernard Lahire "la teoría de los campos no da ninguna herramienta para introducirse ni en las obras, ni en las prácticas, ni en los discursos" (Lahire, 2002: 21). Al igual que Knorr-Cetina a Bourdieu sólo le interesa el proceso de producción de las prácticas científicas. Aún cuando este explique que devela el código de la estructura del campo científico, poco nos dice sobre el producto de la ciencia. El análisis de la teoría de los campos no refiere al discurso de los artículos como producto de la ciencia, es decir, se ignora la base empírica. La producción científica bajo estos marcos conceptúales es, parafraseando a KnorrCetina, "selectivamente extraído, transformado y construido" (Knorr Cetina, 2005: 54)

De cualquier forma habrá que reconocer que el esfuerzo no es una derrota de la investigación, todo lo contrario, muestra dos problemas de la teoría. Las ventaja clara es que al hacer la descripción del fenómeno, hace explicita una estructura de legitimación, poder y prestigio, que sin embargo, nos dice poco sobre los productos de la ciencia. 


\section{Bibliografía}

Bourdieu, Pierre (2003a). La distinción. México, Taurus.

Bourdieu, Pierre (2001). El oficio del sociólogo. México, Taurus.

Bourdieu, Pierre (2000). Cosas dichas. Barcelona, Gedisa.

Bourdieu, Pierre (2003b). El Oficio de Científico. Barcelona. Editorial Anagrama.

Bourdieu, Pierre (2008). Homo Academicus. Argentina. Siglo XXI .

Callon, M., Courtial, J. P. y Penan, H. (1993). Cienciometría. El estudio cuantitativo de la actividad científica: de la bibliometría a la vigilancia tecnológica. Gijón: Trea, 1995.

Collazo-Reyes, F, Luna-Morales, M.E, Russell, J.M y Pérez-Ángona. M.A. (2008). "Publication and citation patterns of Latin America and Caribbean journals in the SCl and SSCl forn 1995 to 2004". Scientometrics, DOI:10.1007/s11192-1841-6.

Crane, Diana. (1972). Invisible Colleges: Diffusion of Knowledge in Scientific Communities. Chicago, IL: University of Chicago Press.

De Nooy, Wouter, Andrej Mrvar y Vladimir Batagelj (2005). Exploratory Social Networks Analysis with Pajek. New York: Cambridge University Press.

De Solla Price, Derek (1973) [1963]. Hacia una Ciencia de la Ciencia. Ariel: Barcelona.

Domenech, Miquel. (1998). Sociología Simétrica. Editorial Gedisa. Argentina.

Kaplan, H, Sadock, B. (2001). Sinopsis de Siquiatría. Editorial Médica Panamericana, S.A.

Hanneman, Robert. Introducción a los métodos del análisis de redes sociales. Departamento de Sociología de la Universidad de California Riverside. http://wizard.ucr.edu/ rhannema/netwprks/text/textindex.html Versión en castellano en http://www. redes-sociales. net/materiales.

Knorr-Cetina, Karin (2005). La Fabricación del Conocimiento: Un Ensayo sobre el Carácter Constructivista y Contextual de la Ciencia. Buenos Aires: Universidad Nacional del Quilmes Editorial.

Macías-Chapula, César. (1998). “Papel de la informetría y de la cienciometría y se perspectiva nacional e internacional", en: Trabajo presentado en el seminario sobre Evaluación de la Producción Científica, realizado en Sao Paolo por el Proyecto SciELO del 4 al 6 de Marzo del 1998. Pp. 25-41.

Manning, Nick. (2000). "Psychiatric diagnosis under conditions of uncertainty: personal disorder, science and professional legitimacy". Sociology of Health and Illness. Vol. 22 No. 5 ISSN 0141-9889

Manning, Nick. (2002). "Actor Networks, policy Networks and personality disorder". Sociology of Health and Illness. Vol.24 ISSN 0141-9889.

Martin, John-Levi (2002). "Power, Authority, and the Constraint of Belief Systems". American Journal of Sociology 107: 861-904.

Molina, J. L., Muñoz, J. M. Y Domenech, M. (2002). "Redes de publicaciones científicas: un análisis de la estructura de coautorías". REDES- Revista Hispana para el análisis de redes sociales. Vol. 1·3.

Moody, James. (2004). "The Structure of a Social Collaboration Network: Disciplinary Cohesion from 1963 to 1999". American Sociological Review, Vol. 6, No. 2 pp 213-238 
Lahire, Barnard. (2002). "Campo, fuera de campo, contracampo". Colección Pedagógica Universitaria. No. 37-38. Francia

Latour, Bruno (1986). Laboratory Life. Princeton University Press. Inglaterra.

Luhmann, Niklas (1999.) La Ciencia de la Sociedad. Universidad Iberoamericana Anthropos, ITESO, México.

Newman, M. E. J. (2001). "The Structure of Scientific Collaboration Networks". Proceedings of the National Academy of Sciencies of the United States of America, Vol. 98, No. 2. Pp.404-409.

Vélez Cuartas, Gabriel (2010). Las redes de sentido de las redes sociales: Un Estudio Cienciométrico. Tesis Doctoral: Ciencias Sociales y Políticas. Universidad Iberoamericana. Disponible en: Revista Redes. http://www.redes-sociales.net/, consultado el 20 de mayo de 2010

http://hospitals. webometrics.info/. Consultada el 12 de mayo de 2010

http://www. webometrics.info/top100_continent_es.asp?cont=latin_america.

Consultada el 12 de mayo de 2010. 